\title{
Edge Reclamation in Position-Sensitive Photomultiplier Tubes
}

\author{
L. Nakae \\ K. Ziock
}

This paper was prepared for submittal to the

Society of Photo-Optical Instrumentation Engineers Conference

Denver, $\mathrm{CO}$

July 12-16, 1999

June 16, 1999

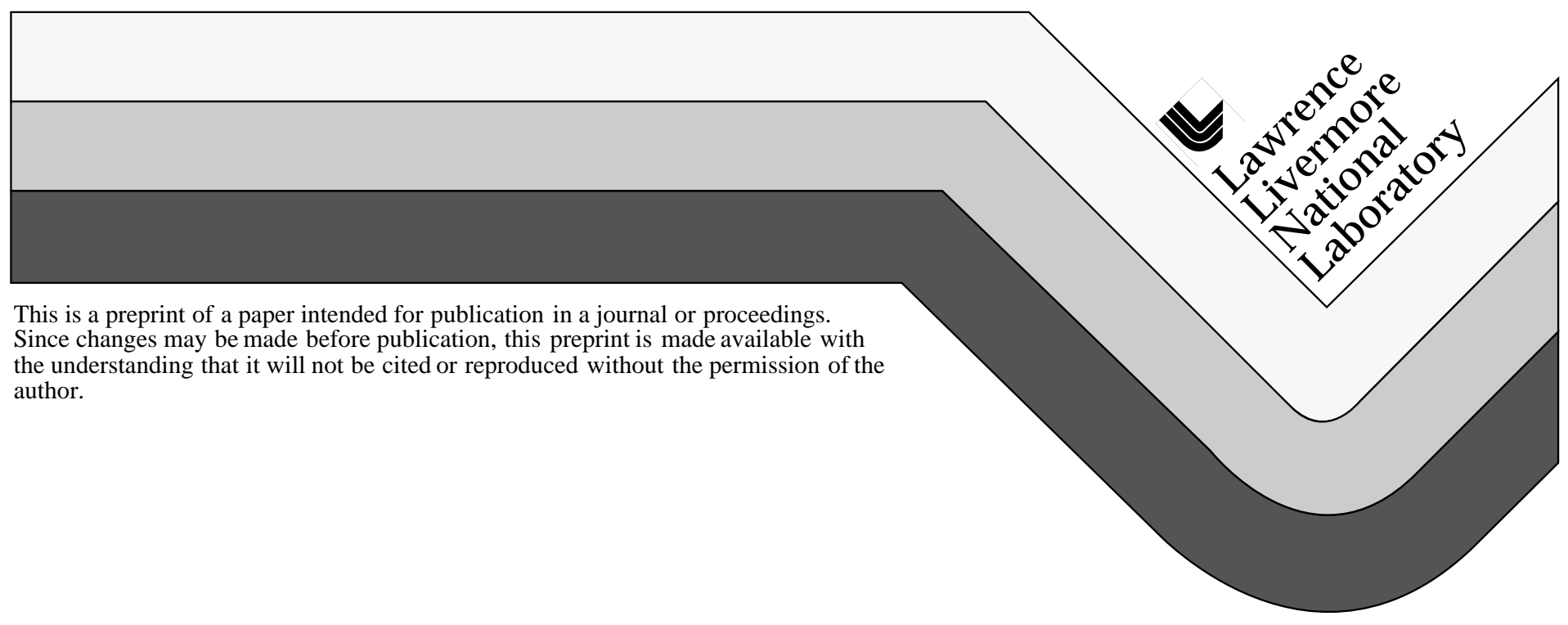




\section{DISCLAIMER}

This document was prepared as an account of work sponsored by an agency of the United States Government. Neither the United States Government nor the University of California nor any of their employees, makes any warranty, express or implied, or assumes any legal liability or responsibility for the accuracy, completeness, or usefulness of any information, apparatus, product, or process

disclosed, or represents that its use would not infringe privately owned rights. Reference herein to any specific commercial product, process, or service by trade name, trademark, manufacturer, or otherwise, does not necessarily constitute or imply its endorsement, recommendation, or favoring by the United States Government or the University of California. The views and opinions of authors expressed herein do not necessarily state or reflect those of the United States Government or the University of California, and shall not be used for advertising or product endorsement purposes. 


\title{
Edge Reclamation in Position-Sensitive Photomultiplier Tubes
}

\author{
L. F. Nakae and K. P. Ziock \\ Lawrence Livermore National Laboratory, L-186, Livermore, CA 94550
}

\begin{abstract}
$\underline{\text { Abstract }}$
We have investigated the performance of a position-sensitive, gamma-ray detector based on a $\quad \mathrm{CsI}(\mathrm{Na})$ scintillator coupled to a Hamamatsu R3292 Position-Sensitive Photomultiplier Tube (PSPMT). The R3292 has an active area 10.0 $\mathrm{cm}$ in diameter (quoted). Utilization of the full active area of the photocathode is a goal that has been previously unrealized due to edge effects. Initial measurements with a $0.75 \mathrm{~cm}$ thick $\mathrm{CsI}(\mathrm{Na})$ crystal indicate that the performance (position resolution linearity) starts to degrade as one reaches a radius of only $3.5 \mathrm{~cm}$, reducing the active area by $60 \%$. Measuring the anode wires we have found that this fall off is not solely due to crystal edge effects, but rather is inherent to the tube crystal system. In this paper we describe the results of our measurements and how good performance can be maintained across a full $10 \mathrm{~cm}$ of the tube face through the use of a few additional electronics channels.
\end{abstract}

\section{$\underline{\text { Introduction }}$}

Position Sensitive Photomultiplier Tubes ( PSPMT's) have been in use by the physics and biomedical communities for many years and are available commercially from Hamamatsu*. The basic construction of PSPMT's consists of crossed anode wires in two planes, each plane connected to a resistive divider chain. The four endpoints of the two chains are read electronically and the resulting signals processed to determine the strength of the signal (sum of the four channels) and the centroid position of the signal on the crossed anode wires. In one dimension (X), a dimensionless position, between 0 and 1, can be found using Angar logic.

$$
\mathrm{X}_{\text {Position }}=\frac{\mathrm{X}^{+}}{\mathrm{X}^{+}+\mathrm{X}^{-}}
$$

where $\mathrm{X}^{+}$and $\mathrm{X}^{-}$are the signals measured from either side of the divider. There is a similar measurement made for the $\mathrm{Y}$ direction. The energy is taken to be the sum of the four channels.

The photocathodes of PSPMT's are predominantly sensitive to visible light but the use of X-ray and gamma-ray sensitive crystals like CsI and BGO or other scintillators coupled to the tube face have made PSPMT's extremely useful for imaging and other applications where positional information of higher energy radiation is required.

In most applications the usable active area of a detector per tube, with minimal cost and weight, is of great importance. However, when coupled with scintillators, the usable area of a PSPMT based detector is considerably less than the area quoted by the manufacturer. In some cases this loss can be as much as $50 \%$. This is due primarily to the loss of light from the edges of the tube and the redistribution of the light through reflection or diffraction to other than its initial position. These effects, which we will refer to generically as "edge effects", work to degrade the position resolution of the tube. In this paper we investigate methods to increase the usable area of a PSPMT.

\footnotetext{
*Hamamatsu Photonics K.K., 314-5, Shimokanzo, Toyooka-village, Iwata-gun, Shizuoka-ken,438-0193, Japan
} 


\section{Light Transport}

We are primarily interested in the detection of $\mathrm{x}$-rays and low energy gamma rays through the use of a scintillator attached to the tube's face. The scintillator will cover the full active area of the PSPMT (all crystals are $12.0 \mathrm{~cm}$ in diameter) and will generally be "thin" with its thickness considerably less than the width $(0.75-1.5 \mathrm{~cm}$ thick). In this application, the "position" of the incoming photon is determined by centroiding a "light cloud" that has been optically transported to the photocathode. We assume that this light cloud is large compared to the anode wire pitch so that centroiding is possible. This results in local energy and position resolutions that are proportional to the square root of the energy (the number of detected photons) [1]. However, the energy resolution is also affected by the absolute number of photons collected from events in different locations and the position resolution is also determined by how accurately the light cloud centroid tracks the gamma-ray interaction location. The edge effects can be understood qualitatively by comparing the behavior of the light reaching the sides of the scintillator and undergoing either perfect reflection or perfect absorption.

Since the scintillation light is emitted into $4 \pi$ steradians more light is incident on surfaces closer to the interaction site. If the light is perfectly reflected at the boundaries (top and sides) and perfectly transmitted through the scintillator and tube, then the total amount of light collected by the tube, at the photocathode, is the same regardless of the position of the incident photon. Consequently, the energy response will be uniform across the entire face of the tube, assuming a uniform photocathode. However even with these idealized conditions the perfect light reflections at the boundary will shift the position of the light cloud's centroid from the real interaction position. This has little or no effect when the gamma ray is incident near the middle of the tube (far from the edges) and an increasing effect as the incident position moves closer to the side of the scintillator. As the incident position of the gamma-ray moves to the edge of the scintillator, the measured centroid position will not properly track the event location and may even, very near the side, become double-valued (the light cloud centroid may even move back toward the center of the tube). This behavior results in poor position resolution of the device and can be seen in figure 1) where the measured position of a collimated source is plotted vs. the true position on the upper part of the figure (scale left). The lower part of the figure shows the 1 sigma widths from a Gaussian fit to the data as a measure of the position resolution (scale right). A perfectly linear tube would appear as a line on the figure. The flattening of the curves at the ends indicate a reduction in centroid motion as the light cloud increasingly interacts with the edge of the detector. This reduction of centroid motion with equal source motion translates into loss of position resolution.

Conversely, if the scintillation light is completely absorbed at the crystal boundaries the measured energy response will vary depending on how much light is lost at the boundaries. The position resolution will also be affected by the loss of light at the boundaries through an increase in statistical fluctuations but the centroid position will still move monotonically with the position of the incident photon. Therefore, both the position and energy resolution will degrade at a given location, because of the loss of light but these changes are overwhelmed by the normal variations in the PSPMT response (e.g. photocathode irregularities) or by the improved ability to determine an event location. However, since the positional information will be single-valued and therefore, in principal, corrections can be made to linearize the response.

\section{Crystal Optimization}

The goal of our work was to modify the PSPMT in such a way as to get the best positional resolution over as large an area as possible while maintaining the best energy resolution. We hoped to obtain this by accentuating the positional dynamic range of the PSPMT near the edges while simultaneously optimizing the light transport, keeping in mind that energy corrections could be made, if necessary, if suitable positional resolution was maintained. The physical attempts to improve positional resolution at the edges could be summarized as attempts to prevent the converted scintillation (visible) light from moving too far from the incident gamma ray position.

We used a Hamamatsu R3292 PSPMT with an $10 . .0 \mathrm{~cm}$ diameter (round) active area. Each of the anode planes has 28 wires connected with $1 \mathrm{k} \Omega$ resistors between them. The CsI (sodium activated) crystal disks (12.0 cm diameter) were coupled onto the tube input window with Bicron BC-630 optical coupling compound. The position and energy 
response of the detector was determined using the $122 \mathrm{keV}$ line of a collimated $\mathrm{Co}^{57}$ source (spot size of $\sim 1.5 \mathrm{~mm}$ ) mounted on computer controlled linear stages. This allowed fine resolution scanning in both directions. We used CsI crystals that were $0.50 \mathrm{~cm}, 0.75 \mathrm{~cm}, 1.0 \mathrm{~cm}, 1.25 \mathrm{~cm}$ and $1.5 \mathrm{~cm}$ thick. Thicker crystals increase the stopping power for higher energy radiation but also increase the size of the "light cloud" on the photocathode and therefore should provide poorer position resolution [1]. Thicker crystals should also increase the area of the "edge effects" since their edges cover a larger solid angle. We investigated the response of a number of physical geometries with primarily the $0.75 \mathrm{~cm}$ thick crystal. Configurations included the bare crystal, the crystal covered with aluminum foil as a reflector, the crystal covered with teflon micro-pore tape (providing diffuse light reflection), blackening the edges of the crystal with black tape (to absorb light reflected from the edges) and various combinations of these materials. We found that the best results were obtained by covering the top of the crystal with teflon tape and the edges with black tape. Figure 1) plots the measured position (in the 0-1 space of Equation (1)) against the known scanned position in mm's (referenced from tube center) of the bare crystal and the improvements from adding the teflon and black tapes to the top and sides of the crystal respectively. We also experimented using a thin aluminum honeycombed grid (roughly $2 \mathrm{~mm}$ hex painted with $\mathrm{Al}$ oxide reflector paint), set in an optical coupling pad, between the CsI crystals and the glass tube face. The geometry was chosen so that the light propagating in the pad at shallow angles to the tube face would be preferentially reflected back toward the center of the light cloud. in order to reduce the spread of the light cloud on the photocathode. Although it showed some promise, so much light was lost to the grid itself that there was an overall loss of positional resolution. A possible better alternative, to grow the CsI directly about such a grid was briefly considered and then discarded as too time intensive and costly for our limited resources.

\section{$\underline{\text { Individual Anode Wire Measurements }}$}

Distortions in the position response of PSPMT's, near the edges of the tubes, have been observed since their inception. Recently Clancy, Thompson, Robar and Bergman [2], addressed this problem and suggested a possible fix that would maintain single-valued positional information over a larger area by altering the resistors on the resistive divider. They measured the signals on individual wires of a Hamamatsu R3941 tube (17x17 wires square) and a segmented BGO crystal and found that the signal had an approximately Gaussian dsitribution across the individual wires. They therefore assumed that the distortion in their divider measurement was due to some signal being lost from beyond the edge of the wires causing an erroneous centroided position from the divider. To compensate for this "lost signal" they increased the resistance on the last step of the divider chain thereby preferentially biasing the signal from the end wires to only one end of the divider. Although this change increased the usable area of the tube, it only provides marginal position resolution in the recovered area. Further, it also had the unwanted effect of worsening the resolution at the center of the tube. This is because the differential change at each point in the divider is fractionally smaller when the resistance at the end of the divider is increased as radically as is needed to have an effect. In the standard divider each step has a $1 \mathrm{k} \Omega$ change from wire to wire so at the middle the step is about 1 part in 8 (for 17 wires) but if one changes the next to the last resistor to $15 \mathrm{k} \Omega$ then the change is only 1 part in 22 . This has the effect of reducing the effective plate scale and therefore the resolution everywhere on the tube.

Since we could not use a detector with poor position resolution in the center, we orginally attempted a modified version of this technique. We altered the ratio of resistances of the first (and last) two resistors of the chain but maintained the sum of the resistances across the entire chain. We did this by making the first resistor $511 \Omega$ and the second resistor $1500 \Omega$. This had the desired effect of boosting the signal from the end wires to their side of the divider extending the linear effect and maintaining the position resolution in the center. However, we found only a slight position improvement away from the center of the PSPMT over the original divider (with the sides blackened and the top covered with teflon tape in both cases). We have opted instead to use a separate readout channel for the end regions of the tube as we describe below.

\section{Single Wire Measurements}

Motivated by the work of Clancy et. al., we measured the signals on individual anode wires both at the center of the phototube and at the edges. We were able to monitor up to 4 anode wires simultaneously and inferred the behavior at other locations as the source position was moved across the instrumented wires. Important differences between our 
configuration and that of Clancy et. al. Includes; 1) the use of a large round PSPMT, 2) the use of continuous nonsegmented crystals and 3) careful termination of the divider ends. The last point is particularly important since we found that a wire in the center of the divider terminated to a low impedance collected significantly more charge than when its impedance to ground matched the values when attached to the complete divider. This indicates that the lowered impedance affects the charge transport to the anode and illustrates that the system is in reality very complex and would benefit from time varying models to fully understand the response. We measured the widths of signals by moving the source across the wires in half $\mathrm{cm}$. steps. At each step, a histogram of ADC counts from each wire from $\sim 10,000$ events was generated and the ADC peak channel determined. The results of these measurements are shown in Figure 2 with channel 1 the wire closest to the right side. We found in the center that the signals were well approximated with Gaussian profiles with a sigma width of roughly $16.5 \mathrm{~mm}$ 's with a $0.75 \mathrm{~cm}$ thick crystal. This Gaussian shaped signal was over a relatively flat background ( $20 \%$ of peak) that is presumably the light multiply reflected internally from all the boundaries which has lost its positional information.

The signals on the wires near the edges are similar to those obtained in the center except for the noticeable difference that the last three wires do not have Gaussian peaks that "turned over" as the source leaves the side of the crystal. In fact, the magnitude of the peaks of the last two wires is suppressed, indicating that these wires collect significantly less charge overall than the other anode wires in the chain even when the source is positioned directly over them. This could be due to the geometric shortening of the wires in our rounded tube as well as the light lost off the side (note we noticed this effect with or without the light absorbing tape on the crystal sides). Figure 3) shows the ADC counts of the last four wires in the divider chain as the source is scanned over them. Here channel 4 is closest to the center of the tube. The degree with which the last two wire peaks are suppressed relative to the inner wires convinces us that no amount of change in the divider resistor chain would be sufficient compensation to linearize the position centroid determination in the tube. We decided instead to measure the end wires separately and see how we could use the information gained to the best advantage.

\section{Divider/End Wire Configuration}

We reconfigured the anode divider chain in the horizontal dimension to exclude a number of wires at each end. The ends of this reduced divider were read in the normal fashion. The extra wires on each end were connected together (separately on each side) and readout with two additional electronics channels. Based on the individual wire measurements described above we first tried two wires connected together at each end, but eventually found that combining three wires improved the performance. Figure 4 shows a schematic for the rewired divider with three wires read separately at the ends. The old impedance of each wire to ground is maintained in the new configuration.

The signals obtained by each of the channels as the source is scanned across the detector face are shown in figure 5). The figure shows the peak of the ADC counts from each channel based on 10000 events. The error bars are the onesigma widths of the peaks, not the uncertainty in the measurement. Channel 1 and 2 are the right and left sides of the new reduced divider. Channels 3 and 4 are the last three wires combined together on the right and the left sides respectively. It is evident upon inspection of the figure that outside of approximately a $30 \mathrm{~mm}$ radius most of the position information is contained in the end channels and a reasonable position determination should be possible. It only remains to determine how to obtain the best position resolution. The simplest way to add in the extra channels is to add in the extra wires with the divider channel from the same side. Labeling the channels as $\mathrm{X}^{-}$and $\mathrm{X}^{+}$to be the channels from the divider and $X_{1}$ and $X_{r}$ to be the channels from the ganged wires on the appropriate sides, the scheme can be seen as:

$$
X_{\text {Position }}=\frac{X_{r}+X^{+}}{X^{-}+X^{+}+X_{1}+X_{r}}
$$

This is equivalent to adding all the charge deposited on the end wires to one side of the divider. A result similar to simply changing the resistors in the divider. The results can be seen in figure 6) plotted as squares and labeled "reduced divider", which shows a modest improvement to the position resolution near the edges over the original 
divider; plotted as circles and labeled "teflon and tape". The position resolution plotted in the figure is derived by calculating the position for each of the $\sim 10000$ events at each source position of a scan. The resulting distribution at each location is fit by a Gaussian profile to obtain a center location and one sigma width. A polynomial (up to $5^{\text {th }}$ order) is fit to the centroid locations as a function of source location. The slope of this curve is used to determine the plate scale $(\mathrm{mm} / \mathrm{pixel})$ of the data and is divided into the fit sigma to determine the one sigma position resolution. This procedure is used with each of the following trial functions.

Although the simple function of equation (2) provides a modest improvement, it clearly does not optimally weight the data. It is evident from a comparison of the signals in figure 5 that one must weigh the data from the end channels more heavily when the signal is nearer the tube edges. Our first attempt at a realistic function was to devise a correction term to equation (2) using the signal measured from the end wires. We considered corrections terms of the form

$$
\text { Correction }=A \frac{X_{r}^{n}-X_{1}^{n}}{\left(X^{-}+X^{+}\right)^{n}}
$$

Where $\mathrm{A}$ is an arbitrary constant and $\mathrm{n}$ an arbitrary integer exponent. Note that this (and even the standard Angar logic of equation (1)) is dimensionless. This is necessary in order to keep the position measurement independent of incident gamma ray energy. It was evident that a linear correction term would require a constant term in front of order 2 or 3 in order to keep the measured position linear with respect to the source position. It was also found that a quadratic correction term $(\mathrm{n}=2)$ worked very well with a constant of 0.5 . Cubic terms did not work out satisfactorily because of the error introduced by cubing the fluctuations in the measured channels. The last set of data shown in Figure 6) shows the position calculated with the reduced divider plus the quadratic correction term. These data are shown as diamonds and are labeled "div + quad". The resulting position resolution is now very linear with respect to source position but the resolution still degrades as one nears the edge of the tube.

It is evident from figure 5 that the best position information available for determining the location of an event near the edge of the tube is from the combined end wires as the center divider channels have double-valued information. However the end wires have virtually no information about the location of an event near the center of the tube. Recognizing this we choose to break the tube into regions. Using the reduced divider for the center, the end wires near the side and a linear combination in the transition region. For the end wires, we used a linear function:

$$
\mathrm{X} \text { side }=\frac{\mathrm{X}_{\mathrm{r}}-\mathrm{X}_{l}}{\mathrm{X}^{-}+\mathrm{X}^{+}}
$$

We implemented this by calculating a position measurement plus a quadratic correction term given by:

$$
X \text { Position }=\frac{X^{+}+X_{r}}{X^{+}+X^{-}+X_{r}+X_{1}}+0.5 \frac{X_{r}^{2}-X_{1}^{2}}{\left(X^{+}+X^{-}\right)^{2}}
$$

Based on a position calculated with this equation, we use the divider (if near the center), the ganged wires (if near the edge), or a linear combination of the two (if somewhere in between). This was accomplished by using the data in figure 5. A polynomial fit was performed separately fitting the known source positions to the measured positions of the reduced divider alone, and for each of the two sides of end wires. The polynomial fit functions were used to generate lookup tables to do the inverse (go from channel measurement back to tube space). The regions were chosen by scanning the data. The center region (divider only) was chosen to be within $2 \mathrm{~cm}$ of the center, middle region (linear combination of divider and ganged wires) was $2 \mathrm{~cm}$ to $4 \mathrm{~cm}$ from center and ganged wires only anything greater than 4 $\mathrm{cm}$ from center. In order to make a smooth transition from each region; the linear combination of the two terms in the middle region was weighted linearly so the divider had more importance nearer to center and the wires nearer to the 
edge. The result of this scheme turned out quite satisfactorily and is shown in figure 7 as circles and is labeled "composite". Again the upper part of the figure shows the position measured (scale left) and the lower part the resolution (scale right). In figure 7 the transformation was performed to take the 0-1 fractional space back into detector space by using the central linear part of the curve to obtain a "plate scale". The position resolution was found to be uniform and linear from the center all the way to $5 \mathrm{~cm}$ radius at the edge of the tube. Here the data are shown compared to the standard resistor divider readout (teflon and black tape) which had poor resolution beyond a radius of $3.5 \mathrm{~cm}$ and virtually no resolution outside of $4.5 \mathrm{~cm}$, this represents a doubling of useful tube area. The composite scheme is also plotted against the divider plus quadratic correction term which while also being quite linear, does not have as good a resolution near the sides of the tube. The data are also shown in table 1.

\section{$\underline{\text { Conclusions }}$}

We have presented a method where four additional electronics channels can be used to increase the usable area of a PSPMT based gamma-ray detector by up to $100 \%$. Further, this additional area shows a position resolution comparable with that obtained at the tube center allowing the use of a thicker crystal. This technique requires a minimal rewiring of the conventional anode wire resistor divider chain so as to read the combined signals of the last 3 wire on each side separately from the reduced size divider. These signals are then used to determine the position when near the edges of the tube. We wish to emphasize that the results reported here were obtained primarily from the central axis of a large round tube. Preliminary measurements at off axis points indicate that we can expect similar performance over the entire tube face. This same technique should be applicable to the smaller tubes (both round and rectangular) manufactured by Hamamatsu, although the number of wires to include in the end channels may differ, as it will for differing crystal thicknesses.

Based on these results we have prepared a final detector design using a $1 \mathrm{~cm}$ thick $\mathrm{CsI}(\mathrm{Na})$ crystal coupled to the PSPMT. We have modified each plane for 4-channel readout per plane and that detector is currently undergoing detailed calibration. We expect to modify our simple one dimensional linearzation scheme that we have presented above to work over the entire tube face in two dimensions. This detector will form the heart of a coded aperture imager for detection of fissile materials in the LLNL Arms Control Program.

This work is performed under the auspices of the U.S. Department of Energy at the Lawrence Livermore National Laboratory under contract No. W-7405-Eng-48.

\section{References}

[1] C.J. Hailey, F. Harrison, J.H. Lupton, K.P. Ziock, "An Inexpensive, Hard X-ray Imaging Spectrometer for Use in Atomic Physics and X-ray Astronomy” Nucl. Inst. Meth. A276, 340 (1989).

[2] R.L. Clancy, C.J. Thompson, J.L. Robar and A.M. Bergman, "A Simple Technique to Increase the Linearity and Field-of-View in Position Sensitive Photomultiplier Tubes", IEEE Transactions on Nuclear Science, June 1997, vol, 44, (no 3):494-8. 


\begin{tabular}{|c|c|c|c|c|c|c|c|c|}
\hline $\begin{array}{l}\text { Stage } \\
\text { Step }\end{array}$ & Old Div & Width & Simple & width & Div + Quad & Width & Composite & Width \\
\hline-50.0 & -35.07 & 4.35 & -39.01 & 3.50 & -45.36 & 4.63 & & \\
\hline-45.0 & -36.14 & 3.52 & -39.68 & 3.23 & -44.72 & 4.19 & -44.58 & 2.69 \\
\hline-40.0 & -35.53 & 3.12 & -37.85 & 3.16 & -40.54 & 3.84 & -40.00 & 2.52 \\
\hline-35.0 & -32.98 & 3.22 & -34.02 & 3.12 & -35.07 & 3.38 & -34.75 & 2.53 \\
\hline-30.0 & -29.46 & 3.04 & -29.66 & 2.99 & -30.00 & 3.11 & -29.82 & 2.62 \\
\hline-25.0 & -24.93 & 3.03 & -24.94 & 2.89 & -24.99 & 2.89 & -25.01 & 2.92 \\
\hline-20.0 & -20.35 & 2.92 & -19.94 & 2.88 & -19.92 & 2.87 & -19.99 & 2.96 \\
\hline-15.0 & & & -15.10 & 2.77 & -15.07 & 2.81 & -15.13 & 2.87 \\
\hline-10.0 & -10.09 & 2.92 & -10.03 & 2.69 & -10.02 & 2.72 & -10.12 & 2.82 \\
\hline-5.0 & & & -4.98 & 2.64 & -4.95 & 2.69 & -5.11 & 2.87 \\
\hline 0.0 & 0.72 & 2.53 & -0.05 & 2.59 & -0.04 & 2.62 & -0.09 & 2.75 \\
\hline 5.0 & & & 4.97 & 2.46 & 4.96 & 2.51 & 5.02 & 2.79 \\
\hline 10.0 & 9.86 & 2.43 & 10.06 & 2.50 & 10.04 & 2.53 & 10.15 & 2.70 \\
\hline 15.0 & & & 14.90 & 2.40 & 14.88 & 2.46 & 15.03 & 2.62 \\
\hline 20.0 & 20.01 & 2.37 & 20.16 & 2.35 & 20.14 & 2.45 & 20.26 & 2.60 \\
\hline 25.0 & 24.79 & 2.35 & 24.95 & 2.34 & 24.69 & 2.46 & 24.98 & 2.79 \\
\hline 30.0 & 29.24 & 2.24 & 29.45 & 2.40 & 29.69 & 2.59 & 29.99 & 2.57 \\
\hline 35.0 & 33.24 & 2.13 & 34.07 & 2.35 & 34.81 & 2.65 & 34.86 & 2.57 \\
\hline 40.0 & 36.56 & 2.11 & 37.93 & 2.34 & 39.94 & 2.95 & 39.64 & 2.75 \\
\hline 45.0 & 38.81 & 2.20 & 40.67 & 2.46 & 45.13 & 3.46 & 45.24 & 2.62 \\
\hline 50.0 & & & 41.05 & 2.64 & 48.35 & 4.09 & 49.61 & 3.03 \\
\hline
\end{tabular}

Table 1) Data translated to Tube Positions (mm). Widths are One Sigma widths.

Flgure 1) Original Divider

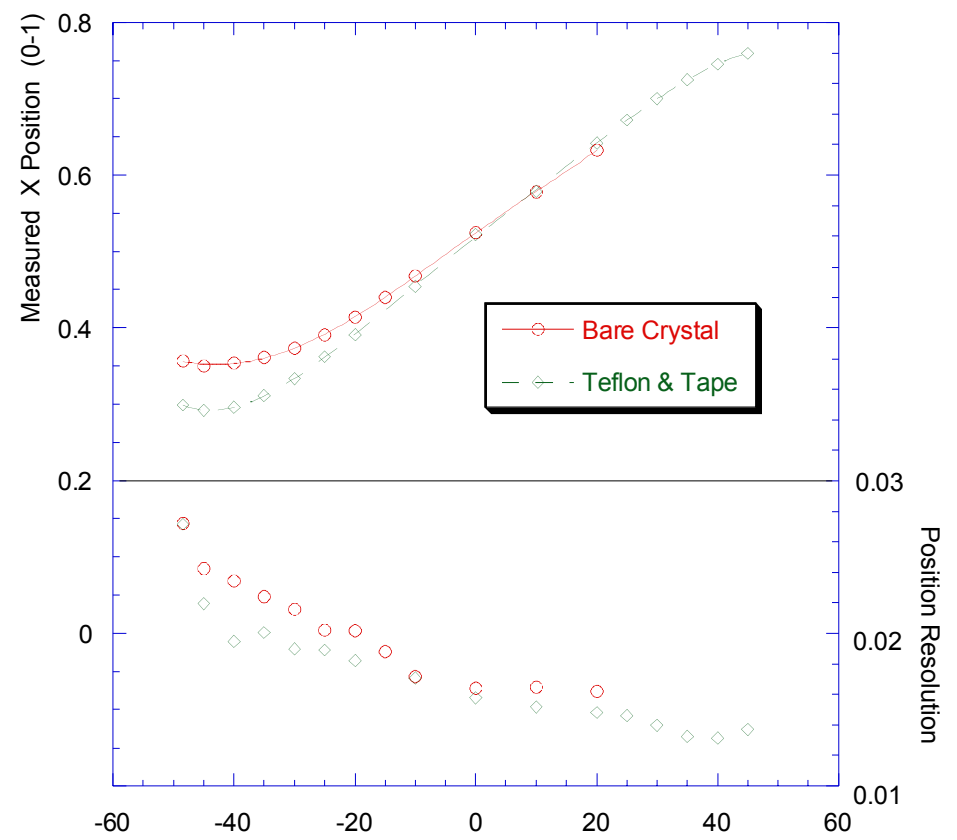

Known Source Position (mm from center) 

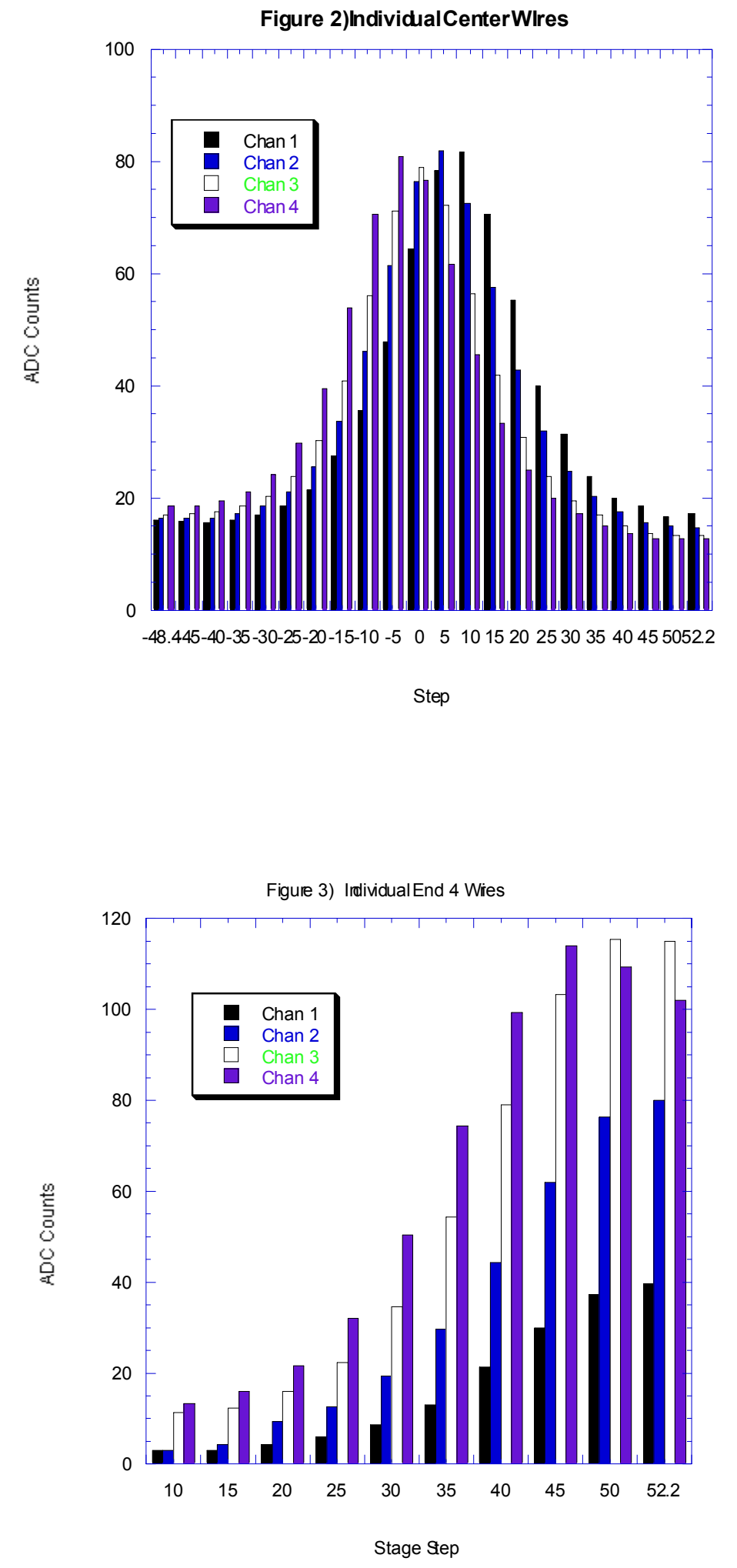


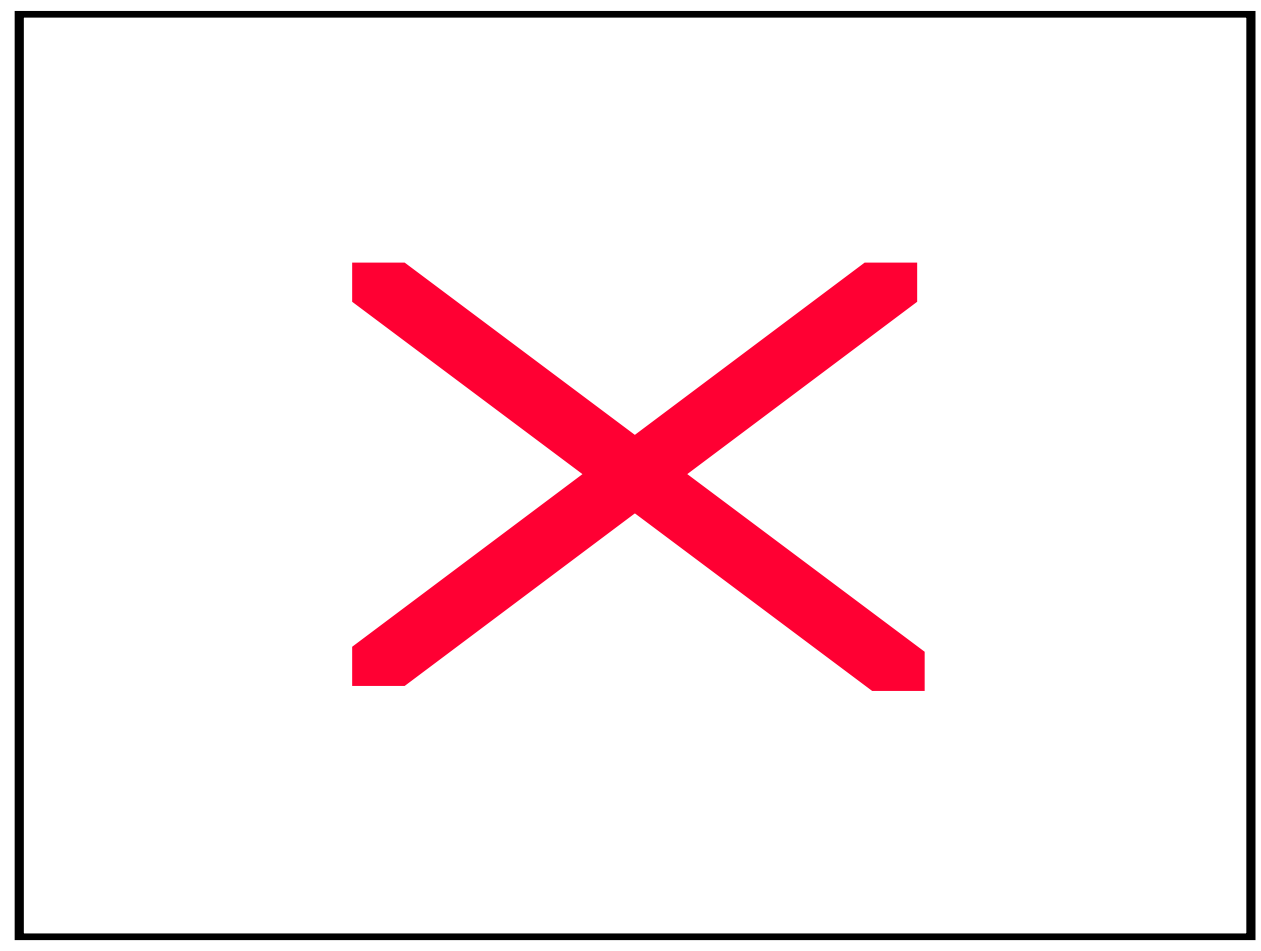

Figure 4) Reconfigured Divider. $R_{0}=1 \mathrm{k} \Omega \mathrm{R} 1=511 \Omega, \mathrm{P} 2=1.5 \mathrm{k} \Omega, \mathrm{R} 3=2.61 \mathrm{k} \Omega$ and $\mathrm{R} 4=3.81 \mathrm{k} \Omega$

Figure 5)Channel Response

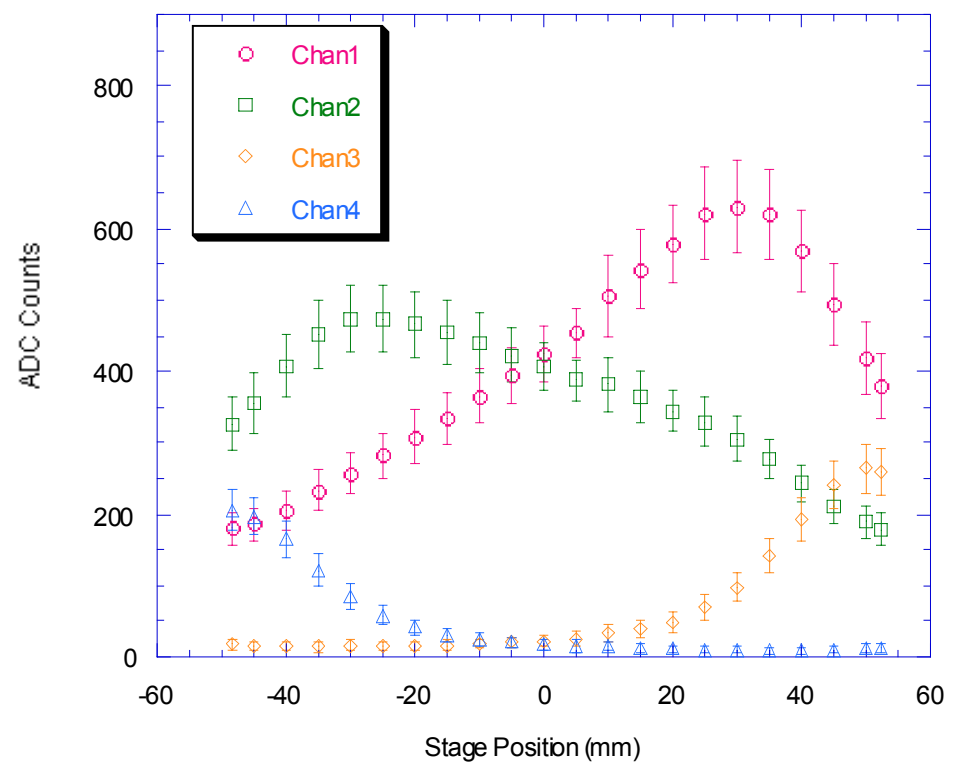


Figure 6) Measured Positions ( fractional space)

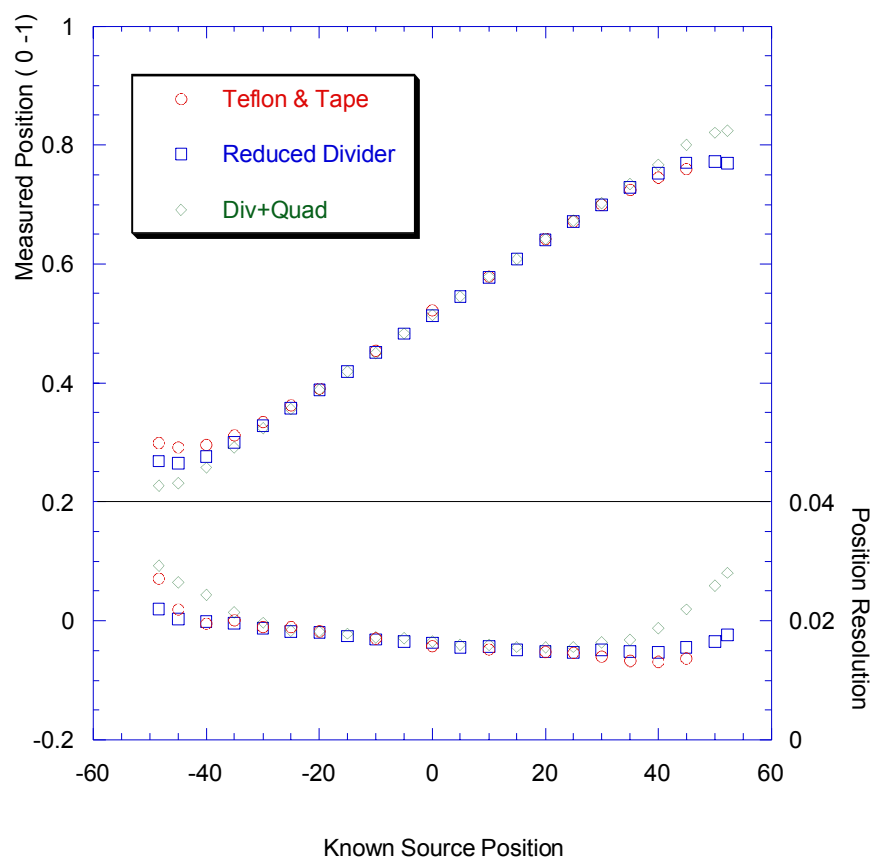

Flgure 7) Measured Positions and Resolutions

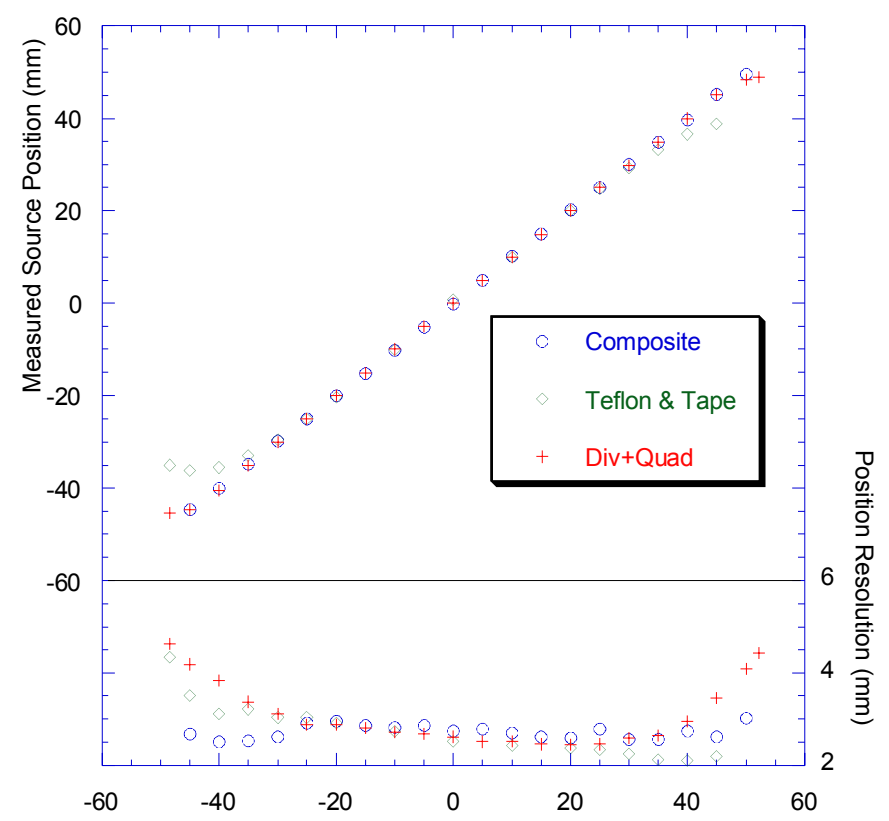

Known Source Position (mm) 